Proyecciones Journal of Mathematics

Vol. 38, No 2, pp. 353-362, June 2019.

Universidad Católica del Norte

Antofagasta - Chile

\title{
Spline collocation approach to study Brachistochrone problem
}

\author{
Pinky M. Shah \\ Veer Narmad South Gujarat University, India \\ and \\ Jyotindra C. Prajapati \\ Sardar Patel University, India \\ Received : November 2017. Accepted : January 2019
}

\begin{abstract}
In this paper authors discussed a problem of quickest descent, the Brachistochrone curve. Spline collocation method is used to solve the non-linear boundary value problem. The numerical results obtained are compared with the transformation method to show effectiveness and accuracy of this method.
\end{abstract}

Subjclass [2010]: 41A15, 37C10.

Keywords: Brachistochrone, optimal control, nonlinear problem, Spline collocation method. 


\section{Introduction}

Johann Bernoulli posed the problem of the Brachistochrone to the readers of ActaEruditorum in June; 1696. He has also published its solution in the next year. After deriving the differential equation for the curve five mathematicians responded with solutions Isaac Newton, Jacob Bernoulli, Gottfried Leibniz, Ehrenfried Walther von Tschirnhaus and Guillaume de IHopital. (Dunham[8]). Solution of the classical Brachistochrone problem using techniques of calculus of variations, (Gelfand and Fomin [11]) or geometrical optics (Erlichson [9]). Problem of finding Brachistochronecurve with coulomb friction lying on a vertical plane in a uniform gravitational field has been discussed by Ashbyet al.[2], Hayen [13], Heijden and Diepstraten [19] and further generalized to a curve with friction lying on a cylinder by Covic and Veskovic[5] and Vratanar and Saje [21] studied related problem of finding the Brachistochrone curve in a non conservative resistance field. Problem of finding Brachistochrone curve on cylinders in uniform fields has been solved by Yamini and Mulhem [22].Brachistochrone curve on spheres mentioned in the unpublished work of Palmieri [14]. A generalization to non-uniform fields has been discussed by several authors (Arvind[1] , Denman [7]). Venezian[20] successfully obtained solution of Brachistochronefor linear radial fields. Parnovsky[15] and Tee[18] got solutions for inverse square redial fields. Further,generalizations of this problem to include specila relativistic effects have been initialed by Farina[10], Goldstein and Bender[12], Scarpello and Ritelli[16]. A study of point-to-point optimal control problem where the objective is to transfer the state of a dynamical system with minimum cost from one point to another point has been discussed by Sussmann and Willem[17]

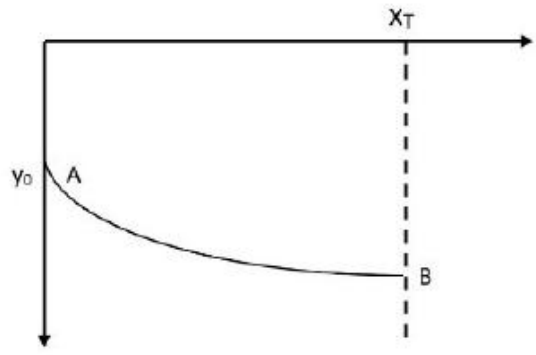

Figure 1. Brachistochrone Problem 
Cal de Boor[6] was first person to introduced bicubic splines method. Bickley[3] brought forward a useful aspect of spline functions in linear two point boundary value problem. In continuation of this study, Blue[4] discussed the applicability of spline functions to non-linear differential equations. In this paper, spline collocation method has been applied to generate approximate solutions of the governing equations of the Brachistochrone problem.

\section{Formulation of Brachistochrone Problem}

Consider two points $\mathrm{A}$ and $\mathrm{B}$ in a space containing a constant gravitational forced field as shown in figure. Point $A$ is the initial point at $\left(0, y_{0}\right)$ a fixed point whereas point $B$ lies anywhere on the terminal line $x=x_{T}$ will the restraint that $\frac{d y}{d x}=0$ at a point. It is the purpose of the methods to find a frictionless path from $A$ to $B$ along which a particle will slide in minimum time. Since gravitational force $I$ the only force acting on the mass, the time of descent $T$ is given by,

$$
T=\int_{0}^{x_{T}} \frac{d s}{V}=\int_{0}^{x_{T}} \sqrt{\frac{1+y^{\prime 2}}{2 g y}} d x
$$

the integrand is defined by

$$
F=\sqrt{\frac{1+y^{\prime 2}}{2 g y}} d x
$$

we get,

$$
T=\int_{0}^{x_{T}} F d x
$$

To minimize the time of descent $T$,Eulers equation is applied to the integrand $F$,

$$
\frac{\partial F}{\partial y}-\frac{d}{d x}\left(\frac{\partial F}{\partial y^{\prime}}\right)=0
$$

Equation (2.4) can be reduce to,

$$
2 y \frac{d^{2} y}{d x^{2}}+\left[1+\left(\frac{d y}{d x}\right)^{2}\right]=0
$$


with boundary conditions,

$$
y(0)=y_{0}, \frac{d y\left(x_{T}\right)}{d x}=0
$$

Equation (2.5) subject to the boundary condition (2.6), is the two point non linear boundary value problem.

Let us introduce following transformations,

$$
\eta=1-\frac{x}{x_{T}}, f=\frac{y}{x_{T}}
$$

then equation (2.5)reduces to,

$$
2 f \frac{d^{2} f}{d \eta^{2}}+\left[1+\left(\frac{d f}{d \eta}\right)^{2}\right]=0
$$

subject to the boundary conditions

$$
\frac{d f(0)}{d \eta}=0, f(1)=3
$$

\section{General Spline Collocation Method}

Use of Spline functions with moments for the solution of nonlinear differential equation was suggested by Blue (1969).

Consider a linear two point boundary value problem

$$
y^{\prime \prime}(x)+p(x) y^{\prime}(x)+q(x) y(x)=r(x)
$$

subject to the boundary conditions

$$
\begin{aligned}
& G_{1}\left[y(a), y^{\prime}(a)\right]=0 \quad \text { at } \quad x=a \\
& G_{2}\left[y(b), y^{\prime}(b)\right]=0 \quad \text { at } \quad x=b
\end{aligned}
$$

Since $s(x)$ is a cubic Spline interpolating $y(x)$ given by equation (3.1),we have $s\left(x_{i}\right)=y\left(x_{i}\right)$, and $s^{\prime \prime}(x)$ is a linear function.

Let us define $s^{\prime \prime}(x)$ in the subinterval $\left[x_{i}, x_{i+1}\right]$ of $[a, b]$ as follows:

$$
s^{\prime \prime}(x)=y_{i+1}^{\prime \prime} \frac{x-x_{i}}{h_{i+1}}+y_{i}^{\prime \prime} \frac{x_{i+1}-x}{h_{i+1}}, i=0,1,2 \ldots, n-1
$$


where $h_{i+1}=x_{i+1}-x_{i}$

On applying two successive integrations on equation (3.4) produced a cubic spline $s(x)$ in $\left[x_{i}, x_{i+1}\right]$ given by

$$
s(x)=y_{i+1}^{\prime \prime} \frac{\left(x-x_{i}\right)^{3}}{6 h_{i+1}}+y_{i}^{\prime \prime} \frac{\left(x_{i+1}-x\right)^{3}}{6 h_{i+1}}+A_{1} \frac{x_{i+1}-x}{h_{i+1}}+B_{1} \frac{x-x_{i}}{h_{i+1}}
$$

where $A_{1}$ and $B_{1}$ are constants to be determined.

Equation (3.5) can be written as

$$
\begin{gathered}
s(x)=y_{i+1}^{\prime \prime} \frac{\left(x-x_{i}\right)^{3}}{6 h_{i+1}}+y_{i}^{\prime \prime} \frac{\left(x_{i+1}-x\right)^{3}}{6 h_{i+1}}+\left(y_{i}-\frac{h_{i+1}^{2}}{6} y_{i}^{\prime \prime}\right) \frac{x_{i+1}-x}{h_{i+1}} \\
+\left(y_{i+1}-\frac{h_{i}^{2}}{6} y_{i+1}^{\prime \prime}\right) \frac{\left(x-x_{i}\right)}{h_{i+1}}
\end{gathered}
$$

Similarly $s(x)$ can be obtained in the interval $\left[x_{i-1}, x_{i}\right]$ as

$$
\begin{gathered}
s(x)=y_{i}^{\prime \prime} \frac{\left(x-x_{i-1}\right)^{3}}{6 h_{i}}+y_{i-1}^{\prime \prime} \frac{\left(x_{i}-x\right)^{3}}{6 h_{i}}+\left(y_{i}-\frac{h_{i}^{2}}{6} y_{i}^{\prime \prime}\right) \frac{x-x_{i-1}}{h_{i}} \\
+\left(y_{i-1}-\frac{h_{i}^{2}}{6} y_{i-1}^{\prime \prime}\right) \frac{\left(x_{i}-x\right)}{h_{i}}
\end{gathered}
$$

From equation (3.5), we obtained $s^{\prime}(x)$ at $x=x_{i}$ denoted by $s^{\prime}\left(x_{i+}\right)$, arrived at

$(3.8) s^{\prime}\left(x_{i+}\right)=-\frac{h_{i+1}}{3} y_{i}^{\prime \prime}-\frac{h_{i+1}}{6} y_{i}^{\prime \prime}+\frac{y_{i+1}-y_{i}}{h_{i+1}}, \quad i=0,1,2, . . n-1$.

and

$$
s^{\prime}\left(x_{i-}\right)=\frac{h_{i}}{6} y_{i-1}^{\prime \prime}-\frac{h_{i}}{3} y_{i}^{\prime \prime}+\frac{y_{i}-y_{i-1}}{h_{i}}, \quad i=1,2, . . n .
$$

Continuity of $s^{\prime \prime}(x)$ at $x=x i$ requires that $s^{\prime \prime}\left(x_{i+}\right)=s^{\prime \prime}\left(x_{i-}\right)$ so that, 


$$
\begin{aligned}
h_{i} y_{i+1}-\left(h_{i}+h_{i+1}\right) y_{i}+h_{i+1} y_{i-1} & =h_{i} h_{i+1}\left(\frac{h_{i}}{6} y_{i-1}^{\prime \prime}+\frac{h_{i}+h_{i+1}}{6} y_{i}^{\prime \prime}+\frac{h_{i+1}}{6} y_{i+1}^{\prime \prime}\right), \\
& (3.10) \quad i=1,2, . . n-1 .
\end{aligned}
$$

Equation $(3.10)$ gives a system of $(n-1)$ equations in $(n+1)$ variables $y_{i}, i=0,1,2, \ldots n$ to be determined. Therefore, the moments $y_{i}^{\prime \prime}, i=$ $0,1,2, \ldots$ can be obtained from equation (3.10) if a curve is initially fitted to the data.

Let us express the equation (3.1) in the form

$$
y^{\prime \prime}(x)=f\left(x, y, y^{\prime}\right)
$$

subject to the boundary conditions (3.2) and (3.3). From these boundary conditions, it can be seen that there are four pairs of boundary conditions as possible combinations viz.

(i) $y(a)=K ; \quad y(b)=L$

(ii) $y(a)=K ; \quad y^{\prime}(b)=L$

(iii) $y^{\prime}(a)=K ; \quad y(b)=L$

(iv) $y^{\prime}(a)=K ; \quad y^{\prime}(b)=L$

For case (iii) equation (3.10) reduces in general form,

$y_{0}-y_{1}=\frac{h_{1}^{2}}{6}\left(2 y_{0}^{\prime \prime}+y_{1}^{\prime \prime}\right)-h_{1} y_{0}^{\prime}$

$h_{i} y_{i+1}-\left(h_{i}+h_{i+1}\right) y_{i}+h_{i+1} y_{i+1}=$

$h_{i} h_{i+1}\left(\frac{h_{i}}{6} y_{i-1}^{\prime \prime}+\frac{h_{i}+h_{i+1}}{3} y_{i}^{\prime \prime}+\frac{h_{i+1}}{6} y_{i+1}^{\prime \prime}\right) . \quad i=1,2,3, . ., n-1$.

$y_{n-2}-2 y_{n-1}=-\frac{h_{n}^{2}}{6}\left(y_{n-2}^{\prime \prime}+4 y_{n-1}^{\prime \prime}+y_{n}^{\prime \prime}\right)-y_{n}$

Similarly, we are able to deal with remaining cases. Now in order to obtain a solution to equation (2.8) with the boundary conditions given in equations (2.9) we fit a straight line $y(x)=m x+c$ through the boundary points, this is an initial guess, the moments are calculated from the relation (3.11) at the nodal points $x=x_{i}$ These moments $y_{i}^{\prime \prime}$ are now utilized to evaluate $y_{i}, i=0,1,2 \ldots n$, through the relation along with the two additional equations from boundary conditions. This can be furnished by solving merely a tridiagonal system of equations. The results so obtained can again be improve by continuing the same process till the desired solutions are found or two successive iterations produce the same values. 


\section{Solution of Brachistochrone Problem by Spline}

Rewrite the differential equation (2.8) in the form of

$$
\frac{d^{2} f}{d \eta^{2}}=-\frac{\left[1+\left(\frac{d f}{d \eta}\right)^{2}\right]}{2 f}
$$

To find the Spline approximation $s(x)$ of $f(\eta)$ described in equation (2.9) satisfying the boundary conditions, a line $f(\eta)=u \eta+v$ is assumed to be the first approximation to start with the iterative scheme. The straight line $f(\eta)=3$ can be fitted through points $x=0$ and $x=1$. The calculation of, $y_{i}, i=0,1,2 \ldots N$ is to be carried out through the solution of tridiagonal system of $(N+1)$ equations. The solution obtained the tridiagonal system of $(N+1)$ equation is given below:

\begin{tabular}{|c|c|c|}
\hline Values of $\eta$ & $\begin{array}{c}\text { Solution using Spline } \\
\text { Method }\end{array}$ & $\begin{array}{c}\text { Solution using Transformation } \\
\text { Method }\end{array}$ \\
\hline 0 & 3.08457122 & 3.0864 \\
\hline 0.092 & 3.083877691 & 3.0763 \\
\hline 0.185 & 3.081796739 & 3.0742 \\
\hline 0.277 & 3.078327057 & 3.0707 \\
\hline 0.369 & 3.073466128 & 3.0658 \\
\hline 0.462 & 3.067210162 & 3.0596 \\
\hline 0.554 & 3.059554079 & 3.0519 \\
\hline 0.646 & 3.050491483 & 3.0429 \\
\hline 0.738 & 3.04001463 & 3.0324 \\
\hline 0.831 & 3.02811439 & 3.0205 \\
\hline 0.923 & 3.014780198 & 3.0071 \\
\hline 1 & 3 & 3 \\
\hline
\end{tabular}

FIGURE 2. Comparison between Spline Solution and Trasformstion Method 


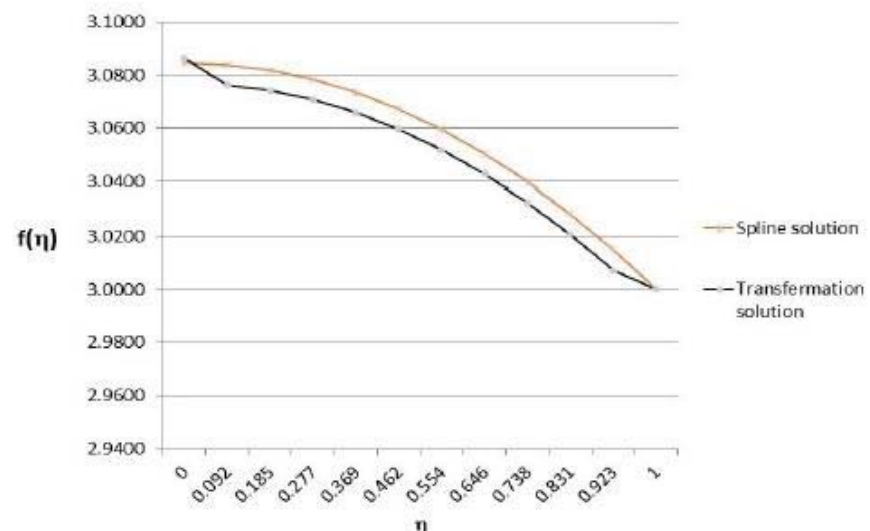

FiguRE 3. Graphical representation of Spline Solution and Trasformstion Method

\section{Conclusion}

The brachistochrone problem can be fit well by an equally space knots. The spline collocation method is considered to find the approximate solution of the brachistochrone problem. An analysis is presented without doing any linearization for solving highly non-linear governing equation. To validate the present system, the result determined by the above analysis has been compared with solution obtained by transform method. The result indicates that spline collocation method gives the best approximation for the non-linear problems without any assumption and linearization. The method is easy to implement and yield very accurate results.

\section{References}

[1] Aravind P. K., Simplified Approach to Brachistochrone Problem, Amer. J. Phys., 49 (9), pp. 884-886, (1981).

[2] Ashby N., Brittin W. E., Love W. F., Wyss W., Brachistochrone with Coulomb Friction, Amer. J. Phys., 43 (10), pp. 902-906, (1975).

[3] Bickley W. G., Piecewise Cubic Interpolation and Two-Point Boundary Problems, The Computer Journal, 11, pp. 206-208, (1968). 
[4] Blue J. L., Spline Function Methods for Nonlinear Boundary Value Problems, Communications of the ACM, 12 (6), pp. 327-330, (1969).

[5] Covic V., Veskovic M. Brachistochrone on a Surface with Coulomb Friction, Int. J. of Nonlinear Mechanics, 43, pp. 437-450, (2008).

[6] Deboor C., Bicubic splines interpolation, J. Math. Phys., 41, pp. 212218, (1962).

[7] Denman H. H., Remarks on Brachistochrone Tautochrone Problem, Amer. J. Phys., 53 (3), pp. 224-227, (1985).

[8] Dunham, William. Journey Through Genius, NewYork: Penguin Books, 1991.

[9] Erlichson H.,Johann Bernoullis Brachistochrone Solution Using Fermats Principle of Least Time, Eur. J. Phys., 20, pp. 299-304, (1999).

[10] Farina C., Bernoulliś Method for Relativistic Brachistochrones, J. Phys. A: Math. Gen., 20, pp. L57-L59, (1987).

[11] Gelfand and Fomin. I. M., S. V. Calculus of Variations. Englewood Cliffs, NJ: Prentice-Hall,Inc., 1963.

[12] Goldstein. H. F., Bender. C. M., Relativistic Brachistochrone, J.Math.Phys.,27, pp. 507-511, (1986).

[13] Hayen J. C.,Brachistochrone with Coulomb Friction, Int. J of NonLinear Mechanics 40, pp. 1057-1075, (2005).

[14] Palmieri, Duzur The Brachistochrone Problem a New Twist to an Old Problem, Undergraduate Honors Thesis, Millers ville University of PA, (1996).

[15] Parnovsky, A. S. Some Generalizations of the Brachistochrone Problem, Acta Physica Polonica, 93, (1998).

[16] Scarpello G. M., Ritelli D., Relativistic Brachistochrone under Electric or Gravitational Uniform Field, Z. Angew. Math. Mech., 86, (9), pp. 736-743, (2006).

[17] Sussmann and Willem: 300 Years of optimal control: From the Brachistochrone to the Maximum principle. Optimal-Control 
[18] Tee G., Isochrones and Brachistochrones, Neural,ParallelSci.Comput., 7, pp. 311-342, (1999).

[19] Vander Heijden. A. M. A., Diepstraten J. D., On the Brachistochrone with Dry Friction, Int. J. Non-Linear Mech., 10, pp. 97-112, (1975).

[20] Venezian G., Terrestrial Brachistochrone, Amer. J. Phys., 34 (8), 701, (1966).

[21] Vratanar B., Saje M., On the analytical solution of the Brachistochrone Problem in a Neo conservative Field, Int. J. Non-Linear Mech., 33 (3), pp. 489-505, (1998).

[22] Yamani H. A., Mulhem A. A., A Cylindrical Variation on the Brachistochrone Problem, Amer. J. Phys., 56 (5), pp. 467-469, (1988).

\section{Pinky M. Shah}

Department of Mathematics, Veer Narmad South Gujarat University, Surat 365005,

India

e-mail : pinkyshah2302@gmail.com

and

\section{Jyotindra C. Prajapati}

Department of Mathematics, Sardar Patel University, VallabhVidyanagar388120

India

e-mail : jyotindra18@rediffmail.com 